\title{
COROTATIONS IN SOME HIGHER-ORDER RESONANCES
}

\author{
S.FERRAZ-MELLO, M.TSUCHIDA and J.C.KLAFKE \\ Universidade de São Paulo, Instituto Astronômico e Geofísico \\ BR-01051-São Paulo, SP, Brasil
}

\begin{abstract}
Some results concerning the resonances $3: 1,4: 1$ and $5: 2$ are given and compared to results published by other authors.
\end{abstract}

\section{Symmetrical corotations}

We say that an asteroid undergoes a corotation when it is simultaneously involved in both a mean-motions and a secular resonance. Let us consider a $(p+q): q$ resonance and introduce the usual variables

$$
\theta=\psi-q \varpi ; \quad \theta_{1}=\psi-q \varpi_{1}
$$

where

$$
\psi=(p+q) \lambda_{1}-p \lambda
$$

$\lambda, \lambda_{1}, \varpi, \varpi_{1}$ are the mean longitudes and the perihelion longitudes of the asteroid and Jupiter, respectively. Exact corotations are defined by the conditions $d \theta / d t=0$ and $d\left(\varpi-\varpi_{1}\right) / d t=0$ (or $\left.d \theta_{1} / d t=0\right)$, considered in the frame of the elliptic restricted three-body problem averaged near the mean-motions resonance. This problem has the symmetrical solutions

$$
\theta=0 \quad(\bmod \pi) ; \quad \varpi_{1}-\varpi=0 \quad(\bmod q \pi)
$$

A theory allowing the orbital elements of these solutions to be obtained, for resonances of any order, has been constructed by the authors (Ferraz-Mello, Tsuchida and Klafke, 1992).

\section{Coherence of the motions}

Motions near a corotation have a coherence property which is unique in noncollisional Celestial Mechanics. Let us show an elementary calculation showing that, in the neighbourhood of corotations the motions cluster about $p+q$ longitude values. Indeed, the motions about one stable corotation center may be given by variational relations like

$$
(p+q) \lambda_{1}-p \lambda-q \varpi=\delta \theta ; \quad \varpi-\varpi_{1}=\delta \varpi
$$

where $\delta \theta$ and $\delta \varpi$ are functions of $t$ with small amplitudes and mean values 0 or $\pi$. When Jupiter is at the perihelion of its orbit (i.e, $\lambda_{1}=\varpi_{1}+2 k \pi, k \in \mathbf{Z}$ ), these equations may be combined to give

$$
\lambda=\varpi_{1}-\frac{q \delta \varpi+\delta \theta}{p}+\left(1+\frac{q}{p}\right) 2 k p \pi .
$$




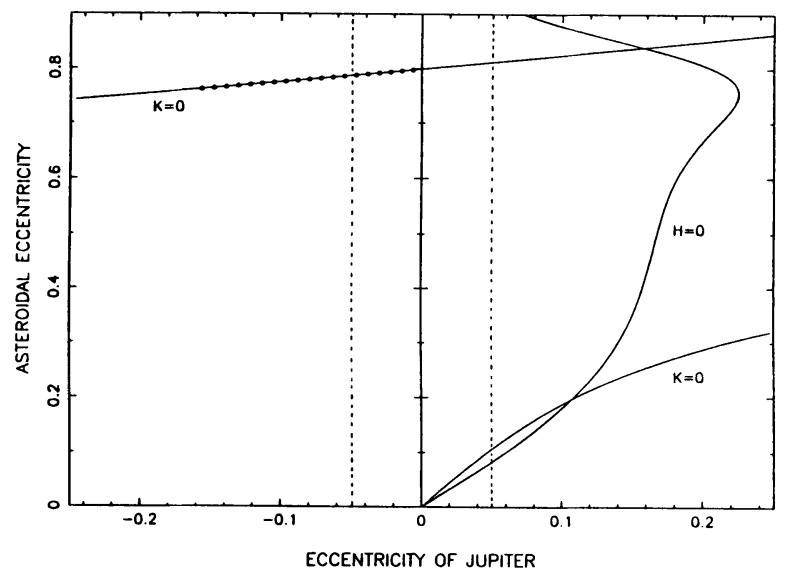

Fig. 1. Locus of the corotation solutions for the resonance $3: 1$. The abcissa is $e_{1} \cos \left(\varpi-\varpi_{1}\right)$ and the ordinate is the asteroidal eccentricity.

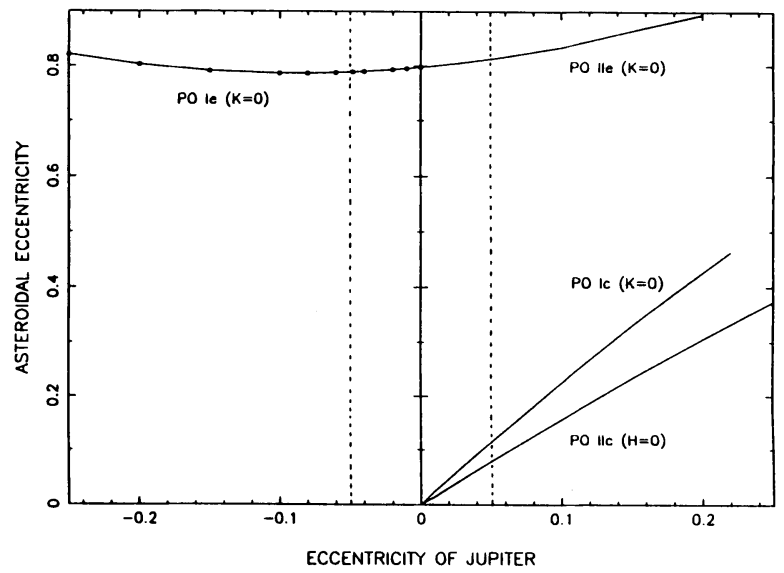

Fig. 2. Families of periodic orbits of the elliptic restricted problem according to Hadjidemetriou (1992) 


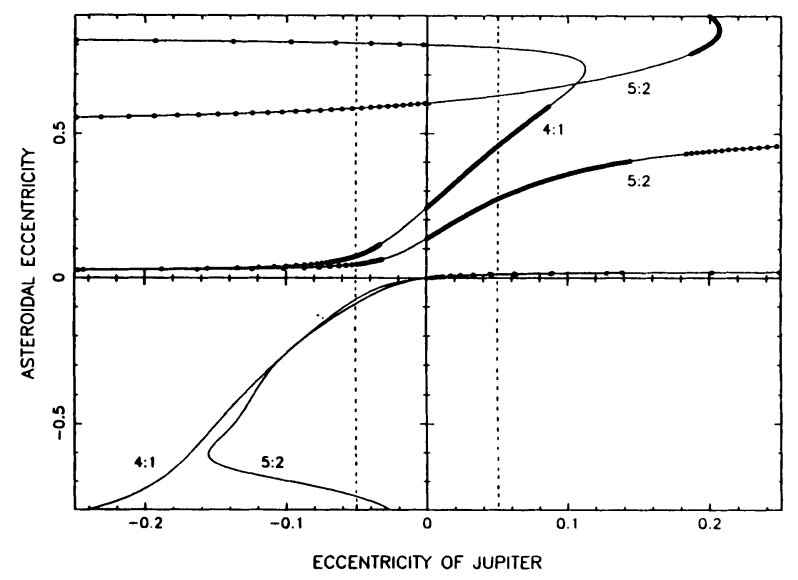

Fig. 3. Locus of the corotation solutions for the resonances $4: 1$ and $5: 2$ (axes as in Fig. 1)

This relation shows that the longitudes of particles in motion about one corotation center cluster about $p$ values $(k=1, \ldots, p)$ and are as close of these values as $\delta \theta$ and $\delta \varpi$ are close to their equilibrium values.

This coherence means that a swarm of particles whose orbital elements are close to a given stable corotation, not only move in similar orbits but, also, cluster about similar longitudes $\left(\bmod 2 \frac{q}{p} \pi\right)$, the particles in each cluster being kept close in space. Corotations play a very important role in the capture of particles in resonances, when a dissipative force causes the evolution of a swarm (see Beaugé and FerrazMello, in these Proceedings).

\section{Results for the resonances $3: 1,4: 1$ and $5: 2$}

The theory has been used to compute the locus of the corotations in the planar planetary case for several resonances.

The corotation solutions for second-order resonances lie in the axes corresponding to $\theta=0$ and $\theta=\pi$. The results for $p=1$ (resonance $3: 1$ ) are shown in Figure 1. The stable-unstable pair of corotation solutions near the bifurcation $(e \approx 0.8)$ and the unstable solution near the origin, in the case of librations about $\theta=\pi$, were also found by Morbidelli and Giorgilli (1990)(see Table I)

The singular points with very-high-eccentricities of the twice averaged Hamiltonian obtained by Yoshikawa (1990) correspond, also, to the stable-unstable pair above mentioned and are located at the positions calculated in this communication. 
TABLE I

Corotation centers according to Morbidelli and Giorgilli (1990); $e_{j u p}=0.05$

\begin{tabular}{clcl}
\hline Resonance & Eccentricity & $\boldsymbol{\pi}-\boldsymbol{p} \boldsymbol{i}_{\text {jup }}$ & Stability \\
\hline $3: 1$ & 0.1 & 0 & unstable \\
& 0.82 & 0 & unstable \\
& 0.79 & $\pi$ & stable \\
$4: 1$ & 0.011 & 0 & unstable \\
& 0.085 & $\pi$ & unstable \\
& 0.45 & 0 & stable \\
$5: 2$ & 0.014 & 0 & unstable \\
& 0.045 & $\pi$ & unstable \\
& 0.27 & 0 & stable \\
\hline
\end{tabular}

Figure 2 shows families of periodic orbits of the elliptic restricted problem computed by Hadjidemetriou (1992), obtained from numerical integrations of the full equations of the motion. The meaning of dots is the same as in figure 1 . The results in this figure may be compared to those of Fig. 1. The differences between the results increase with $\left|e_{1}\right|$ and this is a natural consequence of the limitation of the expansions in our model to $e_{1}^{2}$.

The results for the resonances $4: 1$ and $5: 2$ are shown in Figure 3. These solutions lie in the axis corresponding to $\theta=0 \quad(\bmod \pi)$ and the values corresponding to Jupiter's eccentricity $\mathbf{0 . 0 4 8}$, may be compared to the results of Morbidelli and Giorgilli shown in Table $I$. In this case, the agreement is restricted to higheccentricity solutions. The unstable low-eccentricity solutions found by Morbidelli and Giorgilli appear as stable in Figure 3. This disagreement could be due to the fact that the main terms for third-order resonances, for low eccentricities, are cubic with respect to eccentricities and thus not considered in our model. The comparison to some periodic orbits computed by Hadjidemetriou (personal communication) show good agreement for $e \geq 0.15$.

\section{References}

Ferraz-Mello, S.: 1989, "A semi-numerical expansion of the averaged disturbing function for some very-high-eccentricity orbits", Celest. Mech. 45, 65-68.

Ferraz-Mello, S., Tsuchida, M. and Klafke, J.C.: 1992, "On symmetrical planetary corotations" Celest. Mech. \& Dyn. Astron. (to appear).

Hadjidemetriou, J.D.: 1992, "The elliptic restricted problem at the $3 / 1$ commensurability" Celest. Mech. \& Dyn. Astron. (to appear).

Morbidelli, A., Giorgilli, A.: 1990, "On the Dynamics of the Asteroidal Belt. II. Detailed study of the main resonances", Celest. Mech. Dynam. Astron. 47, 173-204.

Yoshikawa, M.: 1989, "A survey of the motions of asteroids in the commensurability with Jupiter" Astron. Astrophys. $213,436-458$.

Yoshikawa, M.: 1990, Motions of asteroids at the Kirkwood gaps. I. On the 3:1 resonance with Jupiter" Icarus 87, 78-102. 